\title{
A MEGACAM SURVEY OF OUTER HALO SATELLITES. IV. TWO FOREGROUND POPULATIONS POSSIBLY ASSOCIATED WITH THE MONOCEROS SUBSTRUCTURE IN THE DIRECTION OF NGC 2419 AND KOPOSOV 2*
}

\author{
Julio A. Carballo-Bello ${ }^{1}$, Ricardo R. Muñoz ${ }^{1}$, Jeffrey L. Carlin ${ }^{2}$, Patrick Côté ${ }^{3}$, Marla Geha ${ }^{4}$, \\ Joshua D. Simon ${ }^{5}$, AND S. G. DJORgOvski ${ }^{6}$ \\ ${ }^{1}$ Departamento de Astronomía, Universidad de Chile, Camino del Observatorio 1515, Las Condes, Santiago, Chile; jcarball@das.uchile.cl \\ ${ }^{2}$ Department of Physics, Applied Physics and Astronomy, Rensselaer Polytechnic Institute, Troy, NY 12180, USA \\ ${ }^{3}$ National Research Council of Canada, Herzberg Astronomy and Astrophysics, Victoria, BC, V9E 2E7, Canada \\ ${ }^{4}$ Astronomy Department, Yale University, New Haven, CT 06520, USA \\ ${ }^{5}$ Observatories of the Carnegie Institution of Washington, 813 Santa Barbara Street, Pasadena, CA 91101, USA \\ ${ }^{6}$ Astronomy Department, California Institute of Technology, Pasadena, CA, 91125, USA \\ Received 2014 October 14; accepted 2015 March 18; published 2015 May 19
}

\begin{abstract}
The origin of the Galactic halo stellar structure known as the Monoceros Ring is still under debate. In this work, we study this halo substructure using deep Canada-France-Hawaii Telescope wide-field photometry obtained for the globular clusters NGC 2419 and Koposov 2, where the presence of Monoceros becomes significant because of their coincident projected position. Using Sloan Digital Sky Survey photometry and spectroscopy in the area surrounding these globulars and beyond, where the same Monoceros population is detected, we conclude that a second feature, which is not likely to be associated with Milky Way disk stars along the line of sight, is present as a foreground population. Our analysis suggests that the Monoceros Ring might be composed of an old stellar population of age $t \sim 9 \mathrm{Gyr}$ and a new component $\sim 4 \mathrm{Gyr}$ younger at the same heliocentric distance. Alternatively, this detection might be associated with a second wrap of Monoceros in that direction of the sky and might also indicate a metallicity spread in the ring. The detection of such a low-density feature in other sections of this halo substructure will shed light on its nature.
\end{abstract}

Key words: Galaxy: halo - Galaxy: structure - globular clusters: general

\section{INTRODUCTION}

Among the stellar substructures discovered in the Milky Way halo so far, the so-called Monoceros Ring is one of the most challenging structures for Galactic archeology. Unveiled by Newberg et al. (2002) and Yanny et al. (2003) in Sloan Digital Sky Survey (SDSS, York et al. 2000) data as a stellar overdensity at low Galactic latitudes, its nature is still controversial, despite substantial observational efforts (e.g., Conn et al. 2007, 2008; Slater et al. 2014). One of the leading interpretations is that the Monoceros Ring is the remnant of a past accretion event (e.g., Conn et al. 2005; Jurić et al. 2008; Chou et al. 2010; Sollima et al. 2011), similar to that generated by the disruption of the Sagittarius (Sgr) dwarf galaxy, which is orbiting around our Galaxy in an almost polar orbit (e.g., Ibata et al. 1994; Majewski et al. 2003; Bonifacio et al. 2004; Martínez-Delgado et al. 2004; Martin et al. 2004; Bellazzini et al. 2006; Belokurov et al. 2006; Siegel et al 2007; Koposov et al. 2012). In contrast to Sgr, Monoceros lacks a known progenitor system, though it has been proposed and later discarded that the Canis Major overdensity is the accreted system that formed Monoceros (Martin et al. 2004; Momany et al. 2004, 2006; Martínez-Delgado et al. 2005; Bellazzini et al. 2006; Moitinho et al. 2006; Butler et al. 2007; Mateu et al. 2009).

An alternative scenario presents the Monoceros Ring as the result of a distortion of the Galactic plane (Momany et al. 2004, 2006; Hammersley \& López-Corredoira 2011). These studies

\footnotetext{
* Based on observations obtained at the Canada-France-Hawaii Telescope (CFHT), which is operated by the National Research Council of Canada, the Institut National des Sciences de l'Univers of the Centre National de la Recherche Scientifique of France, and the University of Hawaii.
}

suggest that the observed star counts are reproducible considering a flared thick disk without a cut-off at $R \sim 14$ kpc. However, Sollima et al. (2011) have recently shown that none of the available synthetic models for the Milky Way are able to reproduce the observed stellar counts in the Monoceros Ring. Unfortunately, none of the existing arguments favoring or rejecting the extragalactic origin of the Monoceros Ring completely rule out the other hypotheses. Additional processes have been suggested to explain the detection of such a vast halo substructure, including the disk distortion generated by a close encounter (Younger et al. 2008), the existence of caustic rings of dark matter in that position within the Galaxy (Natarajan \& Sikivie 2007) and the accretion of the Sgr dwarf galaxy, which might have a direct impact on the formation of stellar rings in the outer halo (Michel-Dansac et al. 2011; Purcell et al. 2011).

Different spectroscopic studies have reported metallicities for Monoceros in the range of $-1.6<[\mathrm{Fe} / \mathrm{H}]<-0.4$ (e.g., Crane et al. 2003; Rocha-Pinto et al. 2003; Yanny et al. 2003) but recent estimates converged on $[\mathrm{Fe} / \mathrm{H}] \sim-1.0$ with a relatively low dispersion (Ivezić et al. 2008; Conn et al. 2012; Meisner et al. 2012). As for the age of the ring, Sollima et al. (2011) derived a value of $t=9.2 \pm 0.2 \mathrm{Gyr}$ via isochrone fitting in two fields in the anticenter direction.

In this work, we have used deep and wide-field photometry for the globular clusters (GCs) NGC 2419 and Koposov 2 (Kop 2) obtained in the context of a larger photometric survey, to study the stellar populations associated with the Monoceros Ring. NGC 2419 and Kop 2 are located at $d_{\odot}=83.2$ and $\sim 40 \mathrm{kpc}$, respectively (Koposov et al. 2007; Ripepi et al. 2007), in the anticenter region, where an important amount of stellar structures (potentially different from Monoceros) have been found (Grillmair 2006; Grillmair et al. 2008; 
Table 1

Summary of Observations

\begin{tabular}{|c|c|c|c|c|c|c|c|}
\hline Object & $\alpha_{0}(\mathrm{~h} \mathrm{~m} \mathrm{~s})$ & $\delta_{0}(\mathrm{~d} \mathrm{~m} \mathrm{~s})$ & Telescope & Mosaic & $\left\langle X_{g}\right\rangle$ & $\left\langle X_{r}\right\rangle$ & Exp. Time: $g / r(\mathrm{~s})$ \\
\hline Eridanus & $04: 24: 44.50$ & $-21: 07: 42.9$ & CFHT & $1 \times 1$ & 1.33 & 1.33 & $6 \times 270 / 6 \times 270$ \\
\hline NGC 2419 & $07: 38: 08.50$ & $38: 56: 24.9$ & CFHT & $1 \times 1$ & 1.40 & 1.17 & $6 \times 450 / 6 \times 450$ \\
\hline Koposov 2 & 07:58:17.00 & $26: 18: 48.0$ & CFHT & $1 \times 1$ & 1.28 & 1.26 & $6 \times 500 / 6 \times 500$ \\
\hline NGC 7006 & $21: 01: 29.50$ & $16: 14: 45.1$ & CFHT & $1 \times 1$ & 1.01 & 1.01 & $6 \times 240 / 6 \times 240$ \\
\hline
\end{tabular}

Li et al. 2012). The projected positions of the clusters in the sky are consistent with the orbit for the Monoceros ring proposed by the Peñarrubia et al. (2005) model.

\section{OBSERVATIONS}

The data used for this study are part of a larger photometric survey of all outer Galactic halo satellites (R. R. Muñoz et al. 2015, in preparation) carried out with the Megacam imagers at both the Canada-France-Hawaii Telescope (CFHT) in the north and the Magellan II-Clay Telescope in the south. In the particular case of NGC 2419 and Kop 2, the observations were made at CFHT.

Observations with the MegaCam imager on the CFHT were made in queue mode. MegaCam is a wide-field imager consisting of $362048 \times 4612$ pixel CCDs, covering almost a full $1 \times 1 \mathrm{deg}^{2}$ field of view with a pixel scale of $0.187 \mathrm{pixel}^{-1}$. For each object shown in Figure 2 one pointing was observed. For each pointing, six dithered exposures in SDSS $g$ and $r$ in mostly dark conditions were observed, with a typical seeing of $0 . " 7-0 . " 9$. The individual exposure times ranged between 240 and $500 \mathrm{~s}$ in both $g$ and $r$. Table 1 lists a summary of the observing logs for these four objects, including their center coordinates, average air masses in the $g$ and $r$ filters. The dithering pattern was selected from the standard MegaCam operation options in order to cover both the small and large gaps between chips (the largest vertical gaps in MegaCam are six times wider than the small gaps). Point source photometry was carried out using both DAOPHOT/Allstar and ALLFRAME (Stetson 1994) as detailed in Muñoz et al. (2010). The astrometric solutions present in the headers of the images were refined using the freely available $\mathrm{SCAMP}^{7}$ package. Photometric calibration was performed by directly comparing stars in SDSS (DR7) for those objects in the SDSS footprint, and using SDSS fields as secondary standard calibrators for those objects outside.

\section{RESULTS AND DISCUSSION}

\subsection{Color-Magnitude Diagrams}

Around half of the original targets of our primary survey were GCs. Interestingly, NGC 2419, Kop 2, NGC 7006, and Eridanus lie in the predicted direction of the halo substructure known as the Monoceros Ring according to the Peñarrubia et al. (2005) model (Figure 1) and their presence should be revealed by a foreground main sequence (MS) in the respective color-magnitude diagrams (CMDs). Figure 2 shows the CMDs containing both stars within a $5^{\prime}$ radius area and those that are further away from the center of those clusters. Clear MSs are revealed in the CMDs for the surrounding areas of NGC 2419 and Kop 2 in the ranges of $19<g<24$ and $0.3<g-r<1.3$ (right panels). As is clear from the CMDs, the morphology of

\footnotetext{
See http://astromatic.net/software/scamp/.
}

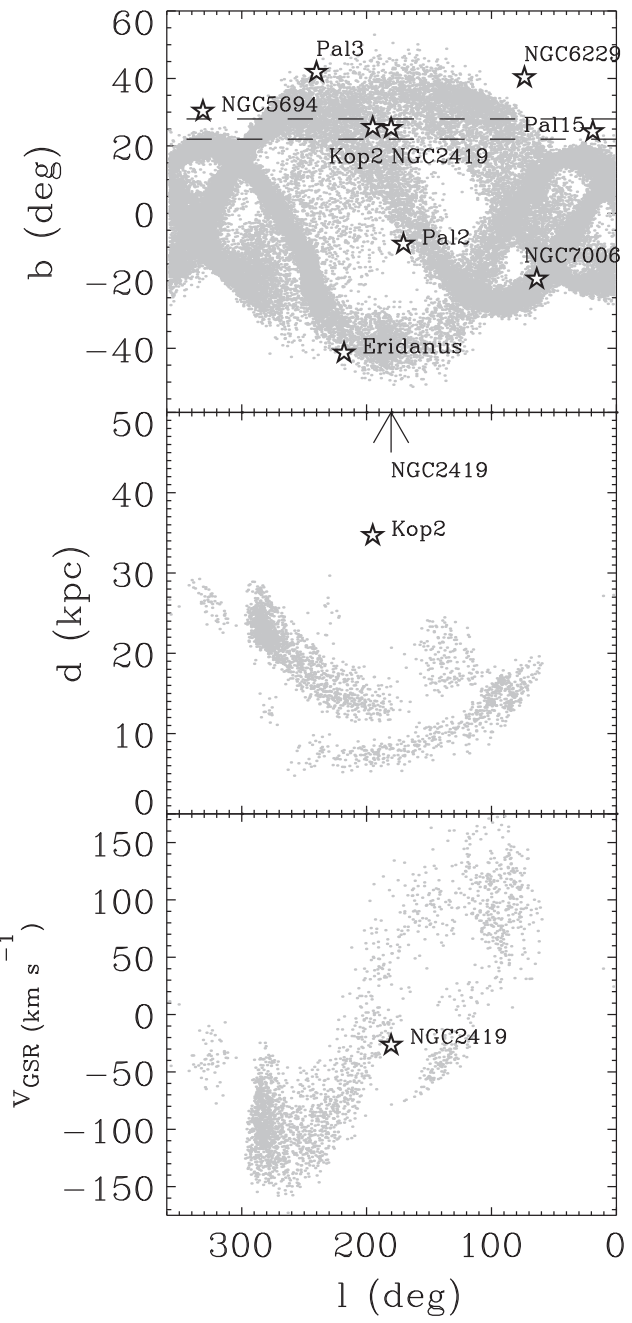

Figure 1. Upper panel: tidal debris spatial distribution of the Monoceros Ring as proposed by the Peñarrubia et al. (2005) model. The position of the GCs included in the Megacam survey and with $|b|<45^{\circ}$ are indicated as stars (Harris 2010). Middle and lower panels: predicted heliocentric distance and velocity (Galactic standard of rest) for the $23<b<28^{\circ}$ section of the ring as defined by the dashed lines in the upper panel, respectively. There is no measured radial velocity for Kop 2 .

these MSs differ from the observed features associated with the GC populations (left panels). These diagrams confirm that NGC 2419 and Kop 2 lie in the same line of sight (LOS) as the vast stellar halo substructure that we identify as the Monoceros Ring. In the case of Eridanus, it is not possible to identify any features that might be associated with a population that are different from those associated with the Milky Way components. Eridanus might still be surrounded by the low surfacebrightness region of Monoceros, but the complete absence of such a substructure is also a possibility. As for NGC 7006, the CMD suggests the presence of an overdensity in its $g>21$ 

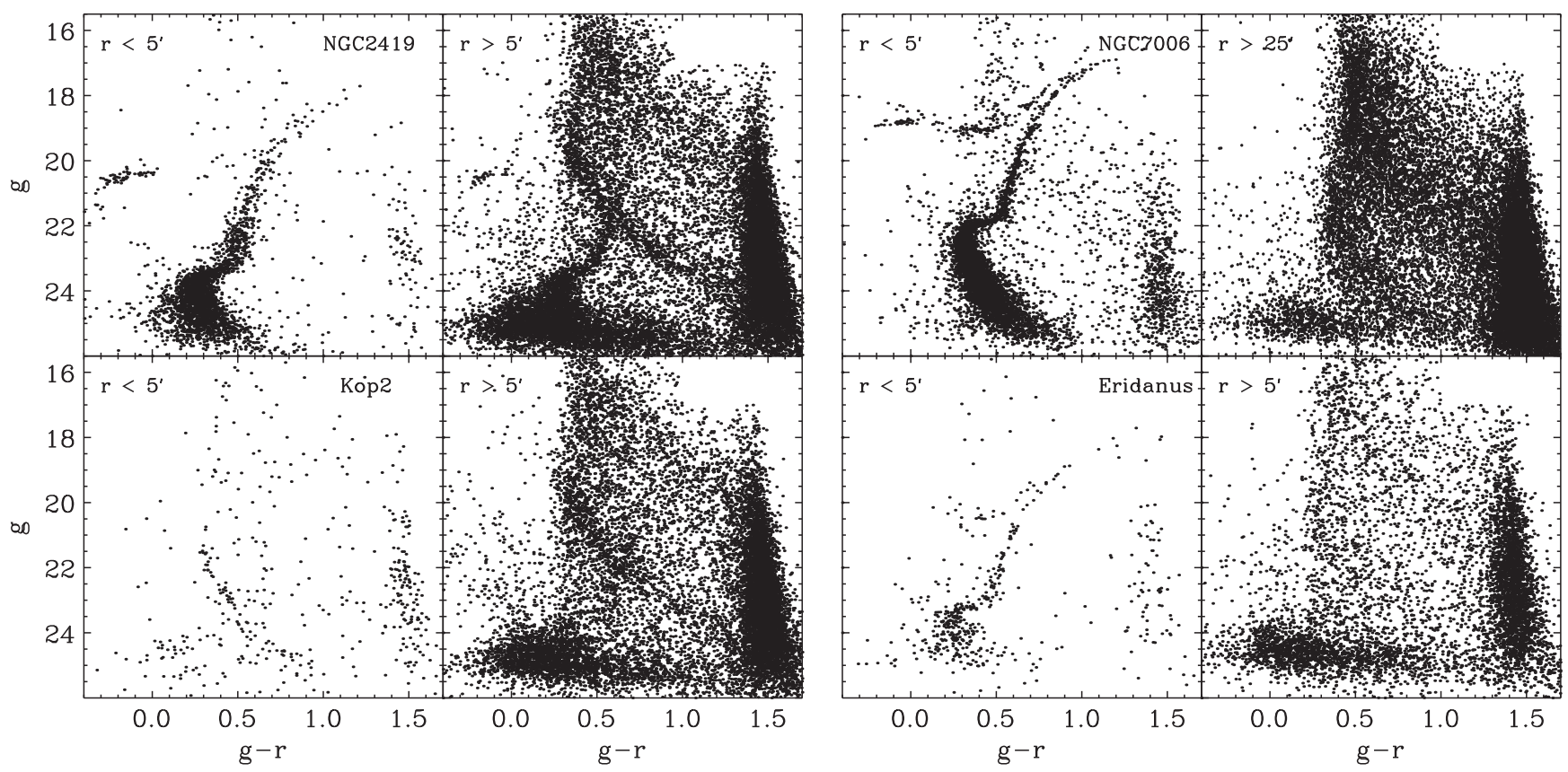

Figure 2. CMDs corresponding to the inner 5 arcmin stellar content and those stars beyond that distance from the centers of NGC 2419 and Kop 2 (left panels) and NGC 7006 and Eridanus (right panels). Note that in the case of NGC 7006, we have selected stars beyond 25 arcmin to reduce the number of field stars. The $r>5$ arcmin CMDs in the cases of NGC 2419 and Kop 2 show the presence of a narrow subjacent MS in the range of $19<g<24$ and are possibly associated with the Monoceros Ring. This feature is not detected in the NGC 7006 and Eridanus diagrams.

section. That subjacent MS might be associated, as proposed by Carballo-Bello et al. (2014), to the presence of stars belonging to the Hercules-Aquila cloud (Belokurov et al. 2007; Simion et al. 2014) along the LOS to the cluster. Alternatively, a higher density of halo stars in that direction of the sky, as suggested by the results of Deason et al. (2014), might produce a broader halo MS as the one observed in our data. In this work, we will focus on the well-defined foreground populations around NGC 2419 and Kop 2 to explore Monoceros.

Since this article does not focus on the clusters themselves, we minimize the number of GC stars in the resulting diagrams, and therefore, while making the CMDs to detect and study Monoceros, we have included only those stars that are away from the cluster's center. The King tidal radii of NGC 2419 and Kop 2 are $r_{\mathrm{t}}=7.5$ and 0.8 , respectively (R. R. Muñoz et al. 2015, in preparation), though this parameter does not necessarily indicate the region beyond which cluster stars are no longer present (see discussion in Carballo-Bello et al. 2012). Thus, to work with the cleanest possible CMDs, we select stars beyond $r=10^{\prime}$ and $3^{\prime}$ from NGC 2419 and Kop 2's centers, respectively.

The (a) and (b) panels of Figure 3 show the resulting CMDs for the area surrounding NGC 2419 and Kop 2, respectively. The observed MS widths indicate the detection of a stellar structure in a narrow distance range along the LOS. We note that the foreground sequences seem to extend blueward of the disk star's turnoff (TO) at $0.20<g-r<0.35$ and $18<g<19$, with a much larger separation, with respect to the tentative Monoceros TO, than the photometric error at this level $(|\delta g| \sim 0.02)$. This feature is observed in both fields, though it is slightly clearer in the NGC 2419 data. To investigate whether this blue population (herein BP) corresponds to the main Monoceros population, we have visually fitted a theoretical isochrone (Dotter et al. 2008) corresponding to the nominal Monoceros age, determined to be $\sim 9$ Gyr by
Sollima et al. (2011) and with a metallicity of $[\mathrm{Fe} / \mathrm{H}]=-1$ (Ivezić et al. 2008; Conn et al. 2012; Meisner et al. 2012). The adopted $E(B-V)$ values in the direction of NGC 2419 and Kop 2 are 0.035 and $0.037 \mathrm{mag}$, respectively (Schlafly \& Finkbeiner 2011). As shown in the (c) panel of Figure 3, this isochrone reproduces the morphology of the foreground MS reasonably well but fails to cover them all the way up to the blue end. By using the region of the isochrone that matches the observed MSs we obtained a radial distance of $d_{\odot}=$ $10.2 \pm 1.6$ and $10.0 \pm 1.5 \mathrm{kpc}$ for the underlying system in the surroundings of NGC 2419 and Kop 2, respectively. These estimates are consistent with the predictions made by the Peñarrubia et al. (2005) model for the Monoceros Ring in that LOS and with the heliocentric distance derived by $\mathrm{Li}$ et al. (2012) for nearby fields on that structure. From the projected position of the clusters in Figure 14 of $\mathrm{Li}$ et al. (2012), it is possible to rule out the so-called anticenter stream discovered by Grillmair (2006) as the subjacent population in the fore/ background of these clusters.

\subsection{SDSS Analysis}

To assess the possibility that these blue extensions of the Monoceros MSs do not represent an actual Monoceros population, but instead correspond to statistical fluctuations of Milky Way stars in this part of the CMD, we have explored an expanded area around the clusters using SDSS data. The right panel in Figure 3 shows all of the objects within a radius of $60^{\prime}$ in five fields in the coordinate range $(\ell, b)=(182,25.35)^{\circ}-(190,25.35)^{\circ}, \quad$ which are located between NGC 2419 and Kop 2. Though SDSS photometry is shallower than our CFHT data, the Monoceros MS is clearly visible. The blue extension observed in the CFHT data is also clear in this CMD.

To establish whether the origin of the BP is Monoceros or Milky Way stars, we study the variation of the BP star counts 


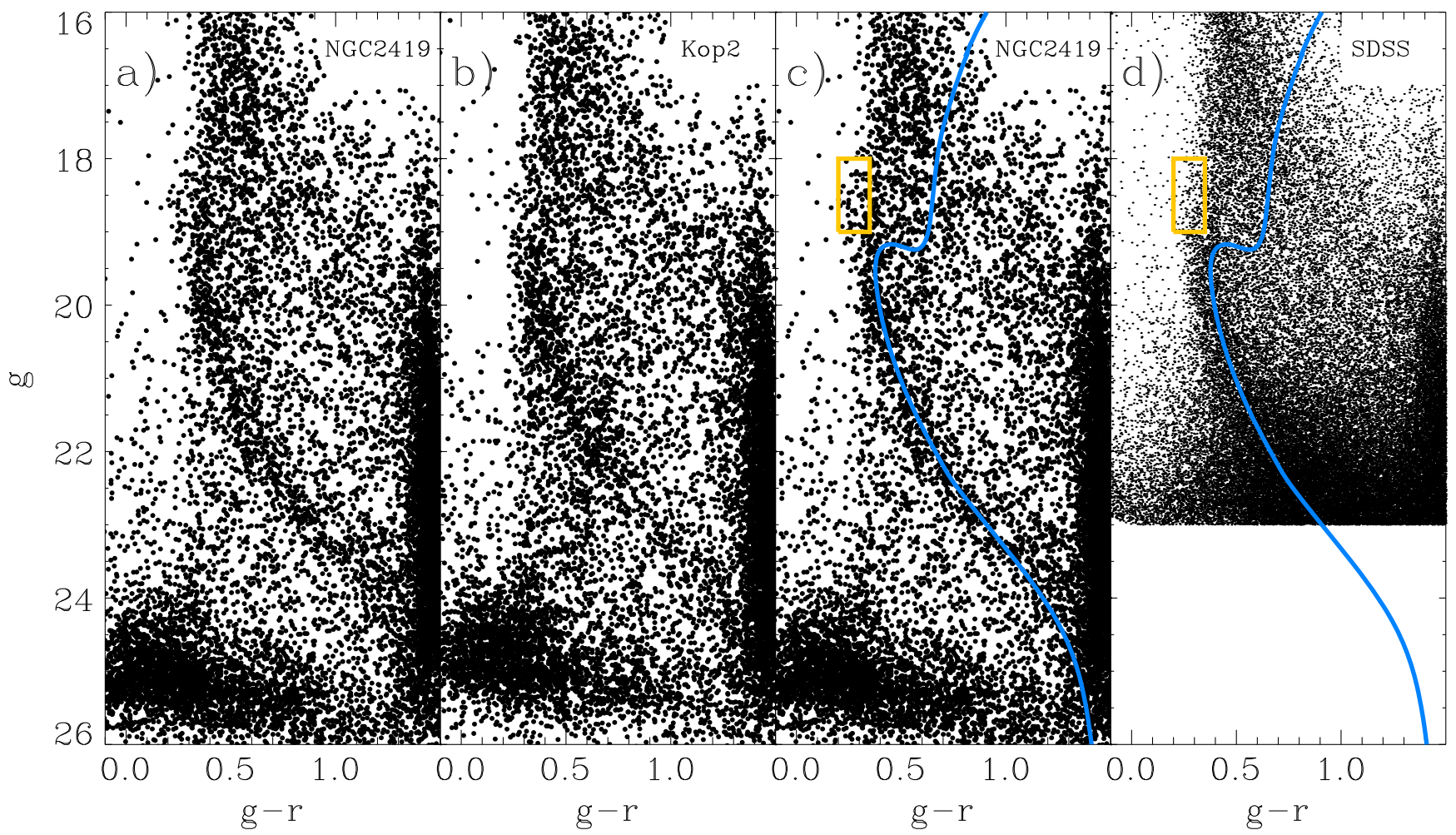

Figure 3. Panels (a) and (b): CMDs corresponding to stars beyond $r=10$ and 3 arcmin from the centers of NGC 2419 and Kop 2 respectively. Panel (c): CMD corresponding to the surroundings of NGC 2419, where the Monoceros isochrone has been overplotted in blue and the position of the BP analyzed in this work is indicated by a yellow box. Panel (d): CMD obtained as the sum of the objects in five SDSS fields between NGC 2419 and Kop 2. Same isochrone and BP position are included for comparison.

with Galactic longitude and compare it to those of a bona fide Milky Way and Monoceros population. To this end, we have defined three regions in the CMD that should predominantly include disk, old Monoceros TO, and BP stars, respectively (see upper panel in Figure 4). We then counted the number of stars in those three regions for 11 SDSS circular fields of $60^{\prime}$ of radius between the coordinates $(l, b)=(180,25)^{\circ}$ and $(\ell, b)=(230,25)^{\circ}$, equidistantly spaced every $5^{\circ}$ in $\ell$. In the bottom panel of Figure 4, we show the gradients in the star counts. To facilitate the comparison between the different boxes, the sequences have been normalized by their value at $\ell=180^{\circ}$. The number of disk stars remains nearly constant for the range of $\ell$ considered while TO and BP counts show a similar behavior. The observed increase in the counts is consistent with the Peñarrubia et al. (2005) model. This result supports the interpretation that the area in the CMD denoted as $\mathrm{BP}$ is not populated by stars associated with the Milky Way disk but by a population possibly associated with Monoceros.

In order to show the morphology of these populations in the CMD with higher contrast, we have compared the SDSS CMDs for those positions that, according to the bottom panel of Figure 4, present a larger difference in density of stars belonging to the ring. In the left panel of Figure 5, we show the Hess diagram corresponding to the CMD, obtained at $(\ell, b)=(210,25)^{\circ}$, when the Milky Way components are removed by subtraction of the CMD corresponding to $(\ell, b)=(180,25)^{\circ}$. The contour of minimal density (lighter gray) indicates the bins with $4 \%$ of the highest density observed. The stellar overdensity above the traditional Monoceros TO has a lower significance with respect to the main ring population, but it clearly confirms the existence of an extra component brighter than the Monoceros TO. The right panel of Figure 5 shows the cumulative luminosity function constructed from the Hess diagram for the color range $0.3<g-r<0.5$. The change of slope in the luminosity function at $g \sim 19.5$ is a possible hint that we are detecting the TO of the older ( $\sim 9 \mathrm{Gyr})$ population, supporting the interpretation that there are multiple populations present in these fields, though we regard this result with caution.

\subsection{Comparison with Synthetic Milky Way Models}

Since it is expected that the regions of the CMDs where Monoceros lies are also populated by Milky Way stars, we estimate the contribution of stars belonging to Galactic components by comparing the observed diagrams with synthetic CMDs generated with the Milky Way photometric models TRILEGAL and Besançon (Robin et al. 2003; Girardi et al. 2005; Vanhollebeke et al. 2009) for the same LOS to each cluster and for a similar solid angle. We have used the set of optimized parameters provided by Gao et al. (2013) as inputs for TRILEGAL, while default values were used for Besançon.

We defined a narrow color range of $0.30<g-r<0.45$ in the CMD to be analyzed and compared with the TRILEGAL and Besançon counts (Figure 6). We counted the number of stars in each of the 15 bins in which the $16.5<g<23$ range was divided, and we compared this number with that obtained for the same color-magnitude bin in the synthetic CMDs. ${ }^{8}$ In the middle panel of Figure 6 , we show the fraction $N_{\text {obs }} / N_{\text {model }}$

\footnotetext{
8 When doing this comparison it was not necessary to correct the observed data for completeness since at the faint magnitude limit of the comparison our photometry is more than $90 \%$ complete.
} 

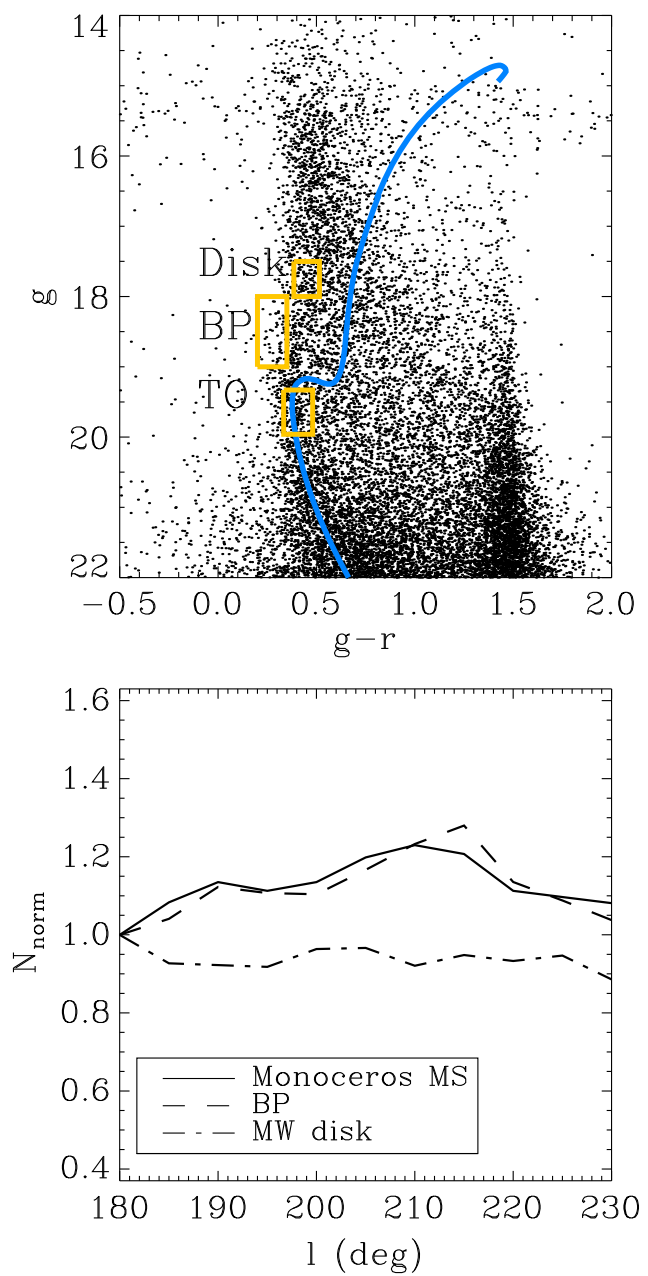

Figure 4. Upper panel: the diagram corresponding to the SDSS field centered at $(l, b)=(184,25.35)^{\circ}$. The overplotted blue isochrone corresponds to the Monoceros Ring. Lower panel: variation of the stellar counts for the three regions defined in the upper $\mathrm{CMD}$ (disk, $\mathrm{BP}$, and TO sample stars indicated as yellow rectangles) as a function of the Galactic longitude in the range of $180<\ell<230^{\circ}$ at $b=25^{\circ}$. All of the sequences are normalized to the first datapoint.

as a function of $g$ magnitude, where $N_{\text {obs }}$ and $N_{\text {model }}$ are the counts obtained for the observed and synthetic CMDs, respectively. With the exception of the range of $18<g<21$, where the presence of Monoceros MS stars is visually dominant, the models considered here adequately reproduce the observed distribution for both the disk and halo stars. The area in the CMD where the contribution of Monoceros stars stands out from the expected stellar counts includes the area defined in this work as BP; however, the significance is lower in comparison with Besançon. Similar results are obtained when we use the surroundings of Kop 2 to carry out these tests.

\subsection{A Younger Population or Metallicity Spread?}

We have determined that the BP corresponds to an actual overdensity and a differentiated population with respect to the Milky Way components. In order to characterize the BP, we used the same family of theoretical isochrones by Dotter et al. (2008), assuming different combinations of age and $[\mathrm{Fe} / \mathrm{H}]$. We considered a grid of possible isochrones with ages between $5-10 \mathrm{Gyr}$ and $-1.6<[\mathrm{Fe} / \mathrm{H}]<-0.8$ to find the best matches to

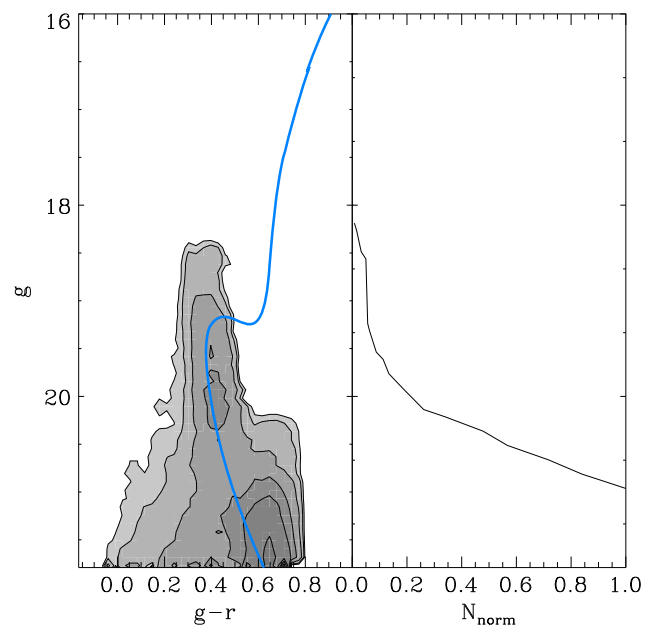

Figure 5. Left: Hess diagram corresponding to the CMD obtained at $(l, b)=(210,25)^{\circ}$, when the Galactic components are removed by the substraction of the CMD corresponding to a field centered at $(l, b)=(180,25)^{\circ}$. The blue isochrone represents the Monoceros Ring stellar population and the minimal density contour indicates the bins with $4 \%$ of the highest density obtained. Right: cumulative luminosity function derived from the Hess diagram and for the color range $0.3<g-r<0.5$. The values have been normalized by the last datapoint considered at $g=22$.

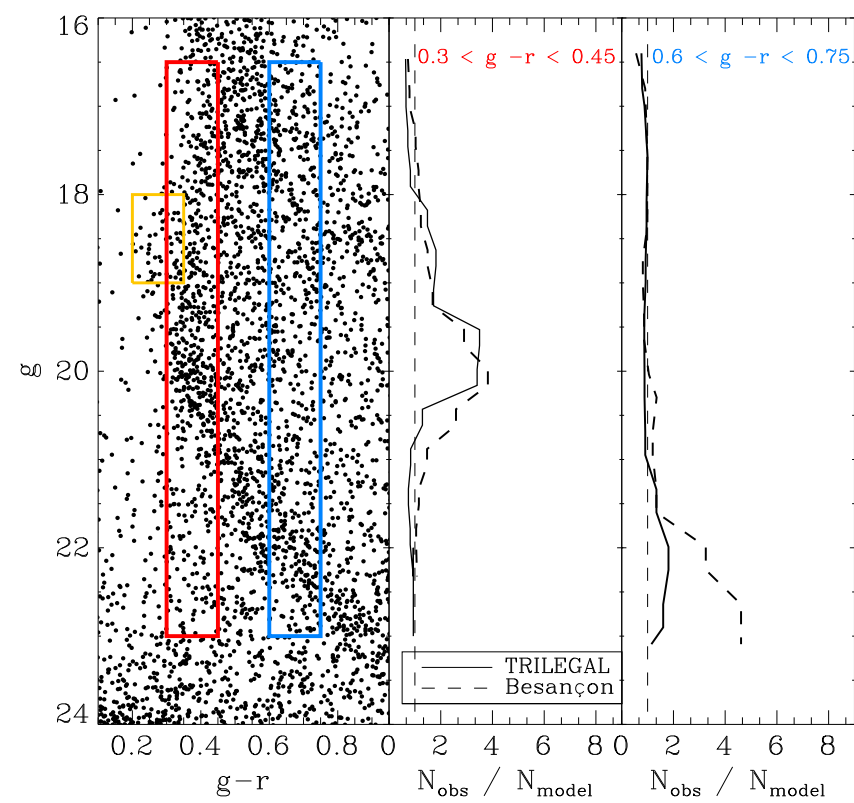

Figure 6. Left: CMD corresponding to the surrounding area of NGC 2419. The red and blue vertical rectangles indicates the color ranges of $0.3<g-r<0.45$ and $0.6<g-r<0.75$, respectively, used for the comparison with TRILEGAL and Besançon. The yellow rectangle shows the position of the suggested BP. Right: $N_{\text {obs }} / N_{\text {model }}$ as a function of $g$ where $N_{\text {obs }}$ and $N_{\text {model }}$ are the counts obtained for the observed and synthetic CMDs, respectively, for the color ranges considered. The solid and dashed lines indicate the results corresponding to TRILEGAL and Besançon.

Monoceros (including the BP region). Reasonable matches are obtained within a range of ages and metallicities (see Figure 7), resulting in distances in the range of $9<d_{\odot}<12 \mathrm{kpc}$. However, the combination that yields a heliocentric distance similar to the one previously derived for Monoceros is an isochrone corresponding to $t \sim 5 \mathrm{Gyr}$ and $[\mathrm{Fe} / \mathrm{H}] \sim-0.95$ (Figure 8). This shows that Monoceros might be composed 


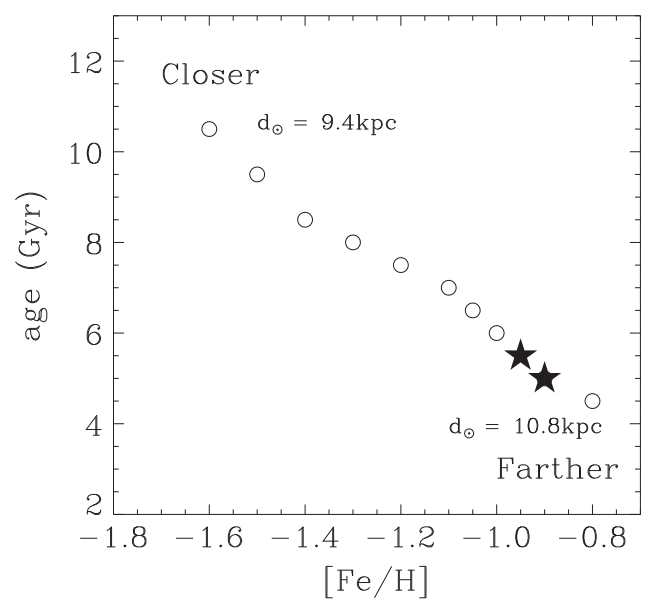

Figure 7. Possible $[\mathrm{Fe} / \mathrm{H}]$-age values for the isochrones that are able to reproduce the $\mathrm{BP}$, including the Monoceros MS up to its faintest end. The filled stars correspond to those combinations resulting in a heliocentric distance compatible with that derived for the Monoceros old population $\left(d_{\odot} \sim 10 \mathrm{kpc}\right)$

of, at least, two stellar populations: a dominant component of $\sim 9$ Gyr and a second contribution of $\sim 4$ Gyr younger. Note that our photometry, where the faintest part of the MS is clearly defined, allows us to discard other possible solutions that would only match the upper MS, a limitation when using shallower data as those from SDSS (see Figure 3 ).

The presence of multiple stellar populations in streams is a natural consequence of the complex properties of their progenitor galaxies. In the case of the Sgr dwarf spheroidal, the presence of old-, intermediate-, and young-aged star formation epochs has been clearly established (e.g., Fahlman et al. 1996; Marconi et al. 1998; Bonifacio et al. 2004; Bellazzini et al. 2006; Siegel et al 2007). In the case of Monoceros, the presence of $\mathrm{M}$ giant stars in the structure around the anticenter (Rocha-Pinto et al. 2003) and the metallicity values reported along different LOSs suggested that possibility. Our detection of a possible younger population in that same region of Monoceros might help establish whether the Monoceros Ring is a complex halo substructure generated by the accretion of a minor satellite or by the distortion of the Galactic disk.

From Figure 7, we conclude that the BP might also be reproduced assuming the isochrone corresponding to a population with a similar age as that of the main Monoceros population but with a lower metallicity of $[\mathrm{Fe} / \mathrm{H}] \sim-1.5$ (see Figure 8). This result agrees with the mean metallicity value obtained by Yanny et al. (2003) and the derived distance $\left(d_{\odot} \sim 9 \mathrm{kpc}\right)$ is consistent within errors with the heliocentric distance for the main Monoceros MS population in that LOS. Therefore, the presence of the BP component in the obtained CMDs might be produced by the manifestation of a metallicity spread suggested by the wide range of values reported for Monoceros in the literature. Alternatively, it is also possible that only a 5 Gyr stellar population is present along this LOS. However, the multiple detections of Monoceros in other areas of the sky have provided an age of $t \sim 9 \mathrm{Gyr}$; therefore, this scenario might be difficult to reconcile with previous work focused on the ring.

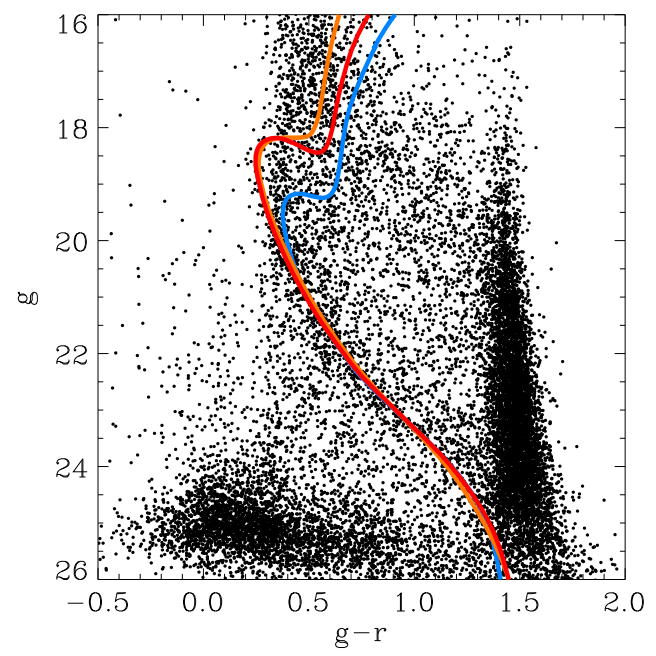

Figure 8. CMD correspondings to the surroundings of NGC 2419. The blue isochrone represents the Monoceros Ring stellar population while the red isochrone indicates the $t \sim 5 \mathrm{Gyr}$ and $[\mathrm{Fe} / \mathrm{H}] \sim-0.95$ population at a similar heliocentric distance as that of the halo substructure. The orange isochrone corresponds to a $t \sim 9 \mathrm{Gyr}$ and $[\mathrm{Fe} / \mathrm{H}] \sim-1.5$ population.

It would be interesting to confirm the presence in other sections of the Monoceros BP using deep wide-field photometry. Even the possible non-detection of hypothetical younger stars in other sections of Monoceros might help us better constrain the location of the progenitor system of this halo substructure, taking advantage of the fact that the different stellar populations are expected to leave the satellite main body at different times (Peñarrubia et al. 2008; Walker 2011).

\subsection{Blue Stragglers}

Since the BP is located blueward and above the TO position of Monoceros, it populates the area of the CMD where blue straggler stars are expected. Recently, Santana et al. (2013) have shown that blue stragglers are ubiquitous among Galactic GCs, classical dwarf spheroidal, and ultra-faint dwarf galaxies. Their analysis shows that in order to reproduce the observed number of blue stragglers in dwarf galaxies, this population should be composed of stars with ages of $t \sim 2.5 \mathrm{Gyr}$ and account for up to the unlikely fine-tuned fraction of $7 \%$ of the total number of stars in the satellite. Despite the fact that the distance uncertainties and depth along the LOS of Monoceros do not allow us to estimate a precise age for the BP, we do not see a significant population of stars extending as far as the MSTO of a $2.5 \mathrm{Gyr}$ old population given by the Dotter et al. (2008) theoretical isochrones. That level is estimated assuming that the brightest members of a blue straggler population should reach a magnitude of $g_{\mathrm{TO}} \sim 16.3$, corresponding to a stellar mass that is twice the mass of a Monoceros MSTO star, at least $\sim 2$ magnitudes brighter than the BP MSTO.

In addition, from our data, we can estimate the specific fraction of blue straggler stars if we assume that the BP is populated entirely by them. Following the procedure described in previous work (e.g., Sollima et al. 2008; Santana et al. 2013), we selected all of the stars in the BP and along the Monoceros MS in the range of $20.5<g<21.5$. After the decontamination of the observed stellar counts, using the synthetic CMD generated with the Besançon model for Section 3.3 as a reference, we conclude that a BP composed of blue stragglers would imply a specific fraction of $F=20 \%$, 
an order of magnitude larger than the observed fraction of blue stragglers in GCs and dwarf galaxies. Therefore, we consider the possibility of the BP being made up entirely of blue straggler stars as unlikely.

\subsection{Foreground Population}

Thus far, we have shown that the BP is likely not associated with a Milky Way disk population and that it is consistent with being part of Monoceros. However, it is still possible that the BP corresponds to a population different from both the Milky Way and Monoceros or to a different wrap of that halo substructure. Given the appearance of the BP in the CMDs, a possibility is that it corresponds to a feature along the LOS between us and Monoceros. If this was the case, we should be able to detect its presence at fainter magnitudes and redder colors, slightly above the well-defined Monoceros MS. To check this scenario, the same comparison with the Milky Way models as in Section 3.3 is performed, but this time in the color range of $0.6<g-r<0.75$. The right panel in Figure 6 shows the results.

In this case, both models reproduce the observed counts with the exception of the $g>21$ range, where we find about four times more stars that are presumably associated with Monoceros than in the synthetic CMD generated with Besançon. The presence of more than one subjacent MS in the redder region of the CMD is not obvious, thus there is not significant evidence in our data of a foreground stream possibly associated with the BP feature.

According to the Peñarrubia et al. (2005) model (Figure 1), two different wraps of Monoceros might be present at different distances along the direction to NGC 2419 and Kop 2 and with indiscernible velocities (see the discussion below). Therefore, our results are compatible with the detection of a different wrap of the ring in that area of the sky; though, the metallicity of such a component would differ from that of the main population of Monoceros observed in these fields (Figure 7). This might support the scenario in which the generation of the $\mathrm{BP}$ is related to the hypothetical Monoceros metallicity spread.

\subsection{Spectroscopic Confirmation of a Second Population in the Line of Sight}

To verify our interpretation that the BP represents a younger sub-population at the same distance as the Monoceros structure, we turn to spectroscopy from SDSS DR10 (Ahn et al. 2014). We select 160 stars around the position of NGC 2419 with colors of $0.2<g-r<0.4$ in the magnitude ranges of $18.0<g<19.0$ for the BP population and 131 stars with $0.25<g-r<0.45$ and $19.25<g<20.0$ for the TO population (see Figure 9). For comparison, we also generate Besançon models in the same field of view and select stars with identical color-magnitude criteria. Figure 10 shows the resulting velocities (left) and metallicities (right) for the BP (upper panels) and TO (lower) in this region. Model predictions are given as blue histograms. A clear excess population is seen in both the $\mathrm{BP}$ and $\mathrm{TO}$ populations at $V_{\mathrm{gsr}} \sim 20 \mathrm{~km} \mathrm{~s}^{-1}$. The velocity dispersion of this structure is clearly much less than the thick disk (which constitutes most of the Besancon stars in the BP selection box), confirming the presence of a kinematically cold structure in both the BP and TO samples, with a velocity compatible with that of the Monoceros Ring in that LOS, as predicted by the Peñarrubia

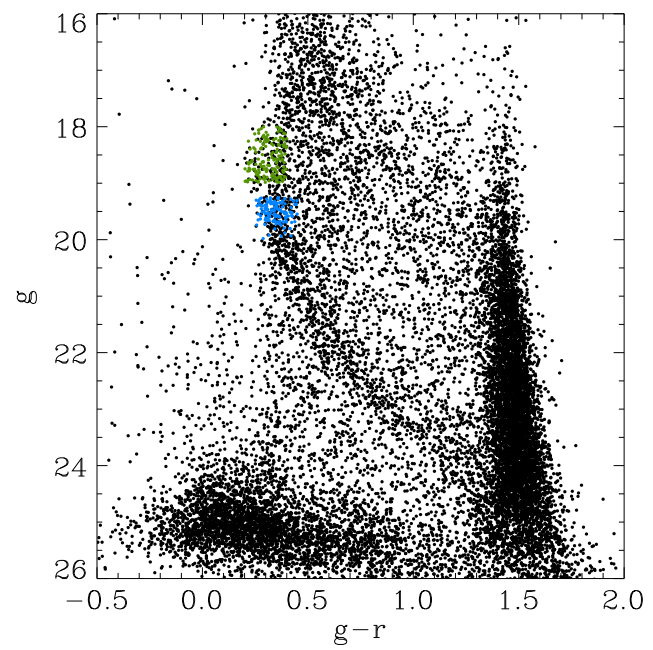

Figure 9. CMD corresponding to the surroundings of NGC 2419. The green and blue points correspond to the position of the BP and Monoceros TO stars used for the analysis of the SDSS spectroscopy data, respectively.

et al. (2005) model (see lower panel in Figure 1). These data are selected in the same sky area as fields denoted "M3" and "M4" by Li et al. (2012), who found a narrow excess at $V_{\mathrm{gsr}} \sim 30 \mathrm{~km} \mathrm{~s}^{-1}$ in field M3, which is likely the same population that we see in Figure 10.

In the right panels of Figure 10, we show the metallicities of stars from each population with $0<V_{\mathrm{gsr}}<50 \mathrm{~km} \mathrm{~s}^{-1}$ to emphasize the velocity substructure. The mean metallicities of the $\mathrm{BP}$ and $\mathrm{TO}$ populations in this velocity range are similar, confirming our finding based on isochrone fitting in Section 3.4.

\section{CONCLUSIONS}

We have used the deep wide-field photometry obtained for the GCs NGC 2419 and Kop 2 to investigate the presence of a second and younger stellar population in the Monoceros Ring. Using SDSS photometry and spectroscopy and the Milky Way synthetic model TRILEGAL, we conclude that there exists a differentiated stellar population that is not associated with any of the other Galactic components and that we have denoted as BP. That population also stands out when we compare it with Besançon, a synthetic model that includes a disk warp and flare.

Isochrone fitting shows that one of the possible explanations for the presence of BP in the CMDs is that Monoceros is composed of an old MS bulk of stars and an additional second population $\sim 4$ Gyr younger, with similar metallicity and lying at a heliocentric distance of $d_{\odot} \sim 10 \mathrm{kpc}$. We have confirmed, using the radial velocities provided by SDSS spectroscopy, that the proposed younger population presents similar kinematics to that of the stellar ring. Alternatively, a metallicity spread might generate a feature as the one observed in the CMD. These results suggest that the hypothetical progenitor galaxy that generated the Monoceros Ring after its accretion might present a more complex composition.

On the other hand, our analysis suggests that a second foreground stellar system along the same LOS might also reproduce the observed CMD morphology. According to the predicted distribution of Monoceros Ring tidal debris, it is possible that this detection corresponds to a second wrap of that substructure and with a different metallicity. 

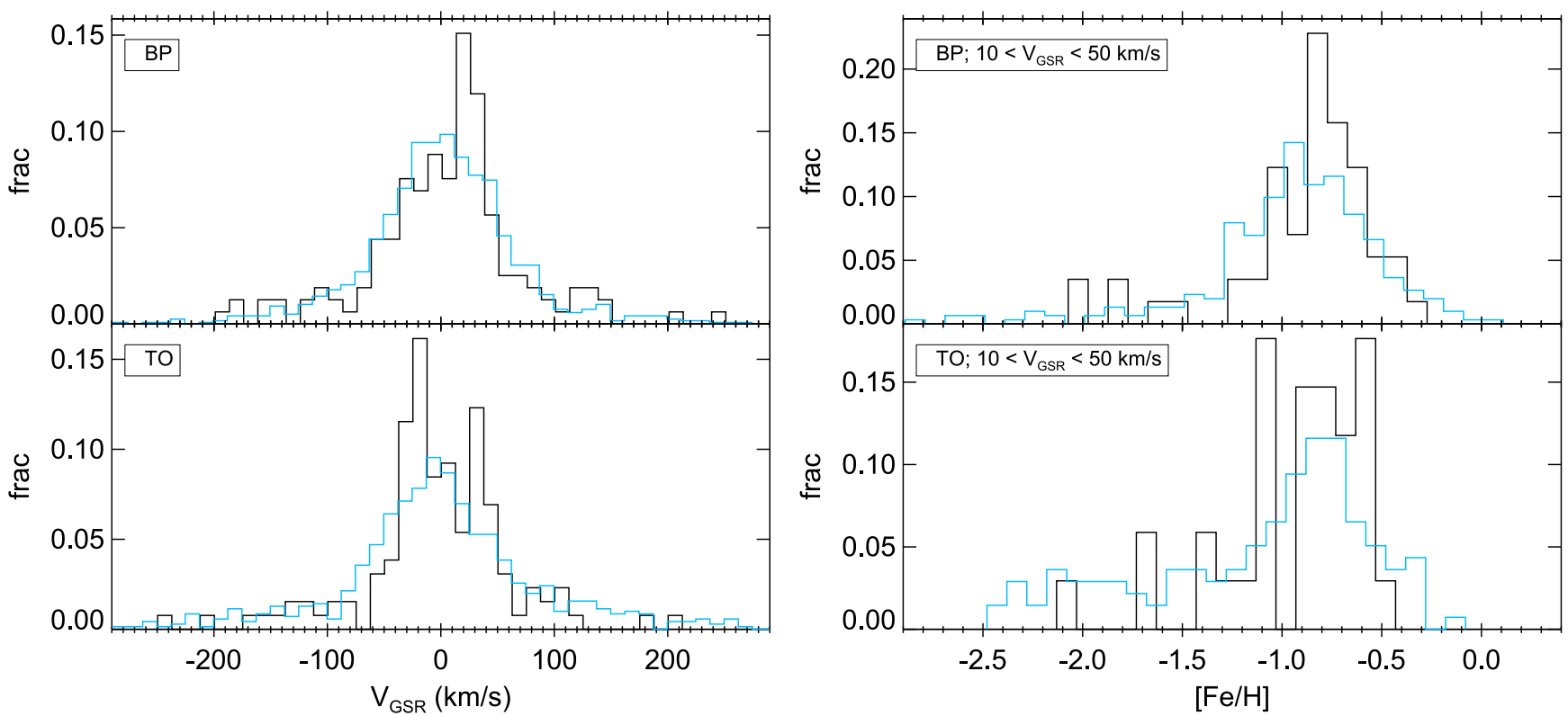

Figure 10. Spectroscopic data from SDSS DR 10 for the region near NGC 2419 (specifically, $175^{\circ}<l<184^{\circ}, 22^{\circ} .7<b<27^{\circ} 7$ ). The upper and lower panels depict velocities (left panels) and metallicities (right panels) of stars with colors of $0.2<g-r<0.4$ in the magnitude ranges of $18.0<g<19.0$ for the BP population and $0.25<g-r<0.45$ and $19.25<g<20.0$ for the TO population. A kinematically cold peak is clearly visible at $V_{\mathrm{gsr}} \sim 20 \mathrm{~km} \mathrm{~s}^{-1}$ in both the BP and TO samples. The right panels show metallicities for only stars between $0<V_{\mathrm{gsr}}<50 \mathrm{~km} \mathrm{~s}^{-1}$. The TO and BP stars present a similar metallicity.

Further deep wide-field photometry of other areas of the sky with high densities of Monoceros Ring stars and at intermediate Galactic latitudes is needed to establish the true nature of the population unveiled in the direction of these two GCs. All of this evidence might shed light on the origin of this controversial halo stellar overdensity.

We warmly thank the anonymous referees for helpful comments and suggestions. J. A. C.-B. and R. R. M. received support from Centre of Excellence in Astrophysics and Associated Technologies (PFB-06). R. R. M. acknowledges partial support from CONICYT Anillo project ACT-1122 as well as FONDECYT project No. 1120013. J.L.C. acknowledges support from NSF grant AST 09-37523. S.G.D. acknowledges partial support from NSF grants AST0909182, AST-1313422, and AST-1413600.

\section{REFERENCES}

Ahn, C. P., Alexandroff, R., Allende Prieto, C., et al. 2014, ApJS, 211, 17 Bellazzini, M., Ibata, R., Martin, N., et al. 2006, MNRAS, 366, 865 Belokurov, V., Zucker, D. B., Evans, N. W., et al. 2006, ApJL, 642, L137 Belokurov, V., Evans, N. W., Bell, E. F., et al. 2007, ApJL, 657, L89 Bonifacio, P., Sbordone, L., Marconi, G., Pasquini, L., \& Hill, V. 2004, A\&A, 414, 503

Butler, D. J., Martínez-Delgado, D., Rix, H.-W., Peñarrubia, J., \& de Jong, J. T. A. 2007, AJ, 133, 2274

Carballo-Bello, J. A., Gieles, M., Sollima, A., et al. 2012, MNRAS, 419, 14

Carballo-Bello, J. A., Sollima, A., Martinez-Delgado, D., et al. 2014, MNRAS, 445, 2971

Chou, M.-Y., Cunha, K., Majewski, S. R., et al. 2010, ApJ, 708, 1290

Conn, B. C., Lane, R. R., Lewis, G. F., et al. 2007, MNRAS, 376, 939

Conn, B. C., Lane, R. R., Lewis, G. F., et al. 2008, MNRAS, 390, 1388

Conn, B. C., Lewis, G. F., Irwin, M. J., et al. 2005, MNRAS, 362, 475

Conn, B. C., Noël, N. E. D., Rix, H.-W., et al. 2012, ApJ, 754, 101

Crane, J. D., Majewski, S. R., Rocha-Pinto, H. J., et al. 2003, ApJL, 594, L119

Deason, A. J., Belokurov, V., Koposov, S. E., \& Rockosi, C. M. 2014, ApJ, 787, 30

Dotter, A., Chaboyer, B., Jevremović, D., et al. 2008, ApJS, 178, 89
Fahlman, G. G., Mandushev, G., Richer, H. B., Thompson, I. B., \& Sivaramakrishnan, A. 1996, ApJL, 459, L65

Gao, S., Just, A., \& Grebel, E. K. 2013, A\&A, 549, A20

Girardi, L., Groenewegen, M. A. T., Hatziminaoglou, E., \& da Costa, L. 2005, A\&A, 436, 895

Grillmair, C. J. 2006, ApJL, 651, L29

Grillmair, C. J., Carlin, J. L., \& Majewski, S. R. 2008, ApJL, 689, L117

Hammersley, P. L., \& López-Corredoira, M. 2011, A\&A, 527, A6

Harris, W. E. 2010, arXiv:1012.3224

Ibata, R. A., Gilmore, G., \& Irwin, M. J. 1994, Natur, 370, 194

Ivezić, Ž., Sesar, B., Jurić, M., et al. 2008, ApJ, 684, 287

Jurić, M., Ivezić, Ž., Brooks, A., et al. 2008, ApJ, 673, 864

Koposov, S. E., Belokurov, V., Evans, N. W., et al. 2012, ApJ, 750, 80

Koposov, S., de Jong, J. T. A., Belokurov, V., et al. 2007, ApJ, 669, 337

Li, J., Newberg, H. J., Carlin, J. L., et al. 2012, ApJ, 757, 151

Majewski, S. R., Skrutskie, M. F., Weinberg, M. D., \& Ostheimer, J. C. 2003 , ApJ, 599, 1082

Marconi, G., Buonanno, R., Castellani, M., et al. 1998, A\&A, 330, 453

Martin, N. F., Ibata, R. A., Bellazzini, M., et al. 2004, MNRAS, 348, 12

Martínez-Delgado, D., Butler, D. J., Rix, H.-W., et al. 2005, ApJ, 633, 205

Martínez-Delgado, D., Dinescu, D. I., Zinn, R., et al. 2004, in ASP Conf. Ser. 327, Satellites and Tidal Streams, ed. F. Prada, D. Martinez Delgado, \& T. J. Mahoney (San Francisco, CA: ASP), 255

Mateu, C., Vivas, A. K., Zinn, R., Miller, L. R., \& Abad, C. 2009, AJ, 137,4412

Meisner, A. M., Frebel, A., Jurić, M., \& Finkbeiner, D. P. 2012, ApJ, 753, 116 Michel-Dansac, L., Abadi, M. G., Navarro, J. F., \& Steinmetz, M. 2011, MNRAS, 414, L1

Moitinho, A., Vázquez, R. A., Carraro, G., et al. 2006, MNRAS, 368, L77

Momany, Y., Zaggia, S. R., Bonifacio, P., et al. 2004, A\&A, 421, L29

Momany, Y., Zaggia, S., Gilmore, G., et al. 2006, A\&A, 451

Muñoz, R. R., Geha, M., \& Willman, B. 2010, AJ, 140, 138

Natarajan, A., \& Sikivie, P. 2007, PhRvD, 76, 023505

Newberg, H. J., Yanny, B., Rockosi, C., et al. 2002, ApJ, 569, 245

Peñarrubia, J., Martínez-Delgado, D., Rix, H. W., et al. 2005, ApJ, 626, 128

Peñarrubia, J., Navarro, J. F., \& McConnachie, A. W. 2008, ApJ, 673, 226

Purcell, C. W., Bullock, J. S., Tollerud, E. J., Rocha, M., \& Chakrabarti, S. 2011, Natur, 477, 301

Ripepi, V., Clementini, G., Di Criscienzo, M., et al. 2007, ApJL, 667, L61

Robin, A. C., Reylé, C., Derrière, S., \& Picaud, S. 2003, A\&A, 409, 523

Rocha-Pinto, H. J., Majewski, S. R., Skrutskie, M. F., \& Crane, J. D. 2003 , ApJL, 594, L115

Santana, F. A., Muñoz, R. R., Geha, M., et al. 2013, ApJ, 774, 106

Schlafly, E. F., \& Finkbeiner, D. P. 2011, ApJ, 737, 103 
Siegel, M. H., Dotter, A., Majewski, S. R., et al. 2007, ApJL, 667, L57

Simion, I. T., Belokurov, V., Irwin, M., \& Koposov, S. E. 2014, MNRAS, 440,161

Slater, C. T., Bell, E. F., Schlafly, E. F., et al. 2014, ApJ, 791, 9

Sollima, A., Lanzoni, B., Beccari, G., Ferraro, F. R., \& Fusi Pecci, F. 2008, A\&A, 481, 701

Sollima, A., Valls-Gabaud, D., Martinez-Delgado, D., et al. 2011, ApJL, 730, L6
Stetson, P. B. 1994, PASP, 106, 250

Vanhollebeke, E., Groenewegen, M. A. T., \& Girardi, L. 2009, A\&A, 498, 95

Walker, M. G., \& Peñarrubia, J. 2011, ApJ, 742, 20

Yanny, B., Newberg, H. J., Grebel, E. K., et al. 2003, ApJ, 588, 824

York, D. G., Adelman, J., Anderson, J. E., Jr, et al. 2000, AJ, 120, 1579

Younger, J. D., Besla, G., Cox, T. J., et al. 2008, ApJL, 676, L21 